\title{
Final focus system tuning studies towards Compact Linear Collider feasibility
}

\author{
E. Marin, ${ }^{*}$ A. Latina, R. Tomás, and D. Schulte \\ CERN, CH-1211 Geneva, Switzerland
}

(Received 7 November 2017; published 19 January 2018; corrected 26 January 2018)

\begin{abstract}
In this paper we present the latest results regarding the tuning study of the baseline design of the final focus system of the Compact Linear Collider (CLIC-FFS). CLIC aims to provide collisions to the experiments at a luminosity above $10^{34} \mathrm{~cm}^{-2} \mathrm{~s}^{-1}$. In order to deliver such luminosity in a single pass machine, the vertical beam size at the interaction point (IP) is reduced to about $1 \mathrm{~nm}$, which imposes unprecedented tuning difficulties to the system. In previous studies, $90 \%$ of the machines reached $90 \%$ of the nominal luminosity at the expense of 18000 luminosity measurements, when considering beam position monitor errors and transverse misalignments of magnets for a single beam case. In the present study, additional static imperfections such as roll misalignments and strength errors are included. Moreover both $e^{-}$and $e^{+}$beamlines are properly simulated. A new tuning procedure based on linear and nonlinear knobs is implemented to effectively cure the most relevant beam size aberrations at the IP. The obtained results for single and double beam studies under solely static imperfections are presented.
\end{abstract}

DOI: 10.1103/PhysRevAccelBeams.21.011003

\section{INTRODUCTION}

The Compact Linear Collider (CLIC) [1] aims to collide $e^{-}$and $e^{+}$at the interaction point (IP), at center-of-mass energy of $3 \mathrm{TeV}$, delivering a nominal luminosity $\left(\mathcal{L}_{0}\right)$ of $5.9 \times 10^{34} \mathrm{~cm}^{-2} \mathrm{~s}^{-1}$ to the experiments. The required transverse beam sizes at the IP $\left(\sigma_{x, y}^{*}\right)$, of the CLIC baseline design, are $40 \mathrm{~nm}$ and $1 \mathrm{~nm}$ in the horizontal and vertical planes, respectively. These nano-beam sizes impose challenging tuning difficulties to the CLIC final focus system (CLIC-FFS) as they scale as $1 / \sigma_{x, y}^{*}$ according to [2].

The CLIC-FFS is based on the local chromaticity correction scheme [3], as the FFS of the International Linear Collider (ILC) $[4,5]$. The local scheme, which has been experimentally demonstrated [6], offers a cost reduction against the non-local scheme, or so-called traditional system, while delivering sufficient luminosity to the experiments.

Studying the tunability of the FFS assuming realistic imperfections is a key ingredient to asses its feasibility. Monte-Carlo simulations are used to sample random initial imperfection configurations. The goal is to reach $110 \%$ of $\mathcal{L}_{0}$ for $90 \%$ of the machines, the $10 \%$ extra margin of $\mathcal{L}$ is set to account for dynamic imperfections. Up to now the CLIC-FFS tuning study has been a simplified version of

\footnotetext{
*emarinla@cern.ch
}

Published by the American Physical Society under the terms of the Creative Commons Attribution 4.0 International license. Further distribution of this work must maintain attribution to the author(s) and the published article's title, journal citation, and DOI. the realistic case, since $e^{-}$and $e^{+}$systems are assumed to be under the same imperfections [7], so called single beam tuning. Indeed single beam tuning is the initial approach to address the tuning. In reality the system is composed of two independent beamlines, double beam study, since ground motion is not yet considered. The results presented in the CLIC Conceptual Design Report (CDR) [7] showed that for the single beam case, $90 \%$ of the machines reach equal or larger $90 \%$ of $\mathcal{L}_{0}$ after 18000 luminosity measurements, when beam position monitor and transverse misalignments of the magnets, were assumed in simulations. An exploratory double beam study [8] assuming the same scale of imperfections as the single case, reveals that only $20 \%$ of the machines reached $\geq 20 \%$ of $\mathcal{L}_{0}$ after only 1700 luminosity measurements, exposing the increased complexity when considering two independent systems.

In contrast, the tuning studies for the ILC-FFS [4] feature a more realistic error scenario as they include transverse and roll alignments errors, strength errors, beam position monitor alignment errors, and ground motion, to mention the most relevant. $90 \%$ of the machines reach $\geq 105 \%$ of $\mathcal{L}_{0}$ for the ILC single beam study. For the double beam case, $90 \%$ of the machines reach $\geq 85 \%$ of $\mathcal{L}_{0}$.

We report on the tuning feasibility of the CLIC-FFS, assuming a more realistic tuning scenario by considering additional static imperfections in both single and double beam cases, while keeping the number of luminosity measurements as low as possible.

In the following sections, we describe our study in terms of considered imperfections, tuning procedure and obtained results in single and double beam tuning studies. 


\section{TUNING STUDY}

The lattice under consideration is the baseline design of CLIC-FFS at center-of-mass energy of $3 \mathrm{TeV}$, the most challenging case. This lattice features a free distance between the IP and the last quadrupole (QD0) equal to $3.5 \mathrm{~m}$, which imposes several challenges to the machinedetector interface, as QD0 sits inside the detector. The lattice can be obtained from [9].

Since the initial error configuration of the machine is unknown, a Monte-Carlo experiment is required to sample various error configurations. For the single and double beam studies, 100 (200) machines with different imperfections are randomly obtained. Each machine is modeled in PLACET [10], our chosen tracking engine, which transports the beam through the CLIC-FFS, taking into account synchrotron radiation.

After assigning the imperfections to the machine elements, the lepton beams are transported from the entrance of each FFS to the common IP. However in the single beam case, only one beam is needed for tracking since its IP particle coordinates are mirrored to represent the opposite beam. The obtained beam distributions at the IP are then handled to the GUINEA-PIG code [11], which computes the luminosity. It should be noted that only the luminosity calculation is used in our tuning procedure based on knobs, since there is no IP beam size monitor.

Once machine imperfections are included in our models the obtained IP beam sizes are of the order of $\mu m$ and thus the luminosity is few orders of magnitude less than $\mathcal{L}_{0}$. In order to reach our tuning goal a tuning procedure is applied to every simulated machine.

\section{A. Imperfections}

The static imperfections assumed in our study are summarized in Table I. These errors are randomly assigned to the beam position monitors and magnets, following a Gaussian distribution of rms $\sigma_{\text {error }}$. Additionally a luminosity calculation error is present due to the limited number of particles used in its evaluation. Since the initial $e^{-}$and $e^{+}$beam distributions are populated with $10^{6}$ particles, the associated error obtained by GUINEA-PIG is below $1 \%$ as shown in Appendix. The initial particle distributions are

TABLE I. List of considered static imperfections included in the past (CDR) and the current studies.

\begin{tabular}{lccc}
\hline \hline & & \multicolumn{2}{c}{$\sigma_{\text {error }}$} \\
\cline { 3 - 4 } Error & Unit & CDR & Present \\
\hline BPM transverse alignment & {$[\mu \mathrm{m}]$} & 10 & 10 \\
BPM roll & {$[\mu \mathrm{rad}]$} & $\cdots$ & 300 \\
BPM resolution & {$[\mathrm{nm}]$} & 10 & 10 \\
Magnet transverse alignment & {$[\mu \mathrm{m}]$} & 10 & 10 \\
Magnet roll & {$[\mu \mathrm{rad}]$} & $\cdots$ & 300 \\
Magnet strength & {$[\%]$} & $\cdots$ & 0.01 \\
\hline \hline
\end{tabular}

assumed to be Gaussian in $x, x^{\prime}, y, y^{\prime}$, and $z$-coordinates and uniform distribution in energy. Also incoming orbit jitter, initial beam mismatched or emittance errors are not considered.

\section{B. Procedure}

The tuning procedure aims to recover the nominal luminosity by correcting the beam orbit by beam-based alignment techniques, aligning the magnets via magnet shunting techniques and removing the IP beam aberrations by scanning a set of precomputed orthogonal knobs. The Brent [12] and Simplex [13] minimization algorithms are used to scan the mentioned knobs one at a time or altogether, respectively. The Simplex algorithm is employed as an alternative optimization method, to countermeasure for potential coupling between the knobs. Although the Simplex algorithm is not as fast as the Brent's one in single knob scan, it allows for multiple knob scan, which in case of lack of orthogonality would equally or better correct the aberrations in less measurements. It should be mentioned that the Simplex approach is not as robust as the parabolic fit against noisy signals.

Beam orbit and luminosity are the observables used for conducting beam-based procedures and knob scan, respectively. In the following the prescription for tuning the single beam case is described in detail. The procedure implemented for the double beam case follows the same strategy as the single beam procedure, with few differences as explained in Sec. II B 2.

\section{Single beam}

The tuning algorithm consists of different steps, being beam-based alignment (BBA) techniques the first one. 1-to-1 [14] and dispersion-free-steering (DFS) [15] correction techniques are applied with all nonlinear magnets switched off. After flattening the orbit and reproducing the dispersion profile at its best, the nonlinear magnets are switched on one-by-one starting from the entrance of the FFS, to conduct their alignment by using the shunting technique $[16,17]$. Next, the DFS process is repeated but now the nonlinear magnets are set to their nominal strengths. The square of the RMS beam obit deviation with respect to the nominal orbit and the square of the RMS dispersion deviation are our figure of merits during 1-to-1 and $D F S$ respectively. After the second DFS correction the calculated luminosities range from $10^{32}-10^{33} \mathrm{~cm}^{-2} \mathrm{~s}^{-1}$ therefore it can be used for tuning in the following steps.

At this stage the obtained $\sigma_{x, y}^{*}$ from the tracking simulations are of the order of few hundreds of nm and few tens of $\mathrm{nm}$ in the horizontal and vertical plane, respectively. Looking at the particle distribution aberration content, one finds that waist shift, coupling and dispersion dominate the observed IP beam size growth. Thus a set of linear knobs based on transverse displacements of the normal sextupole magnets present in the FFS for chromaticity correction, as described 

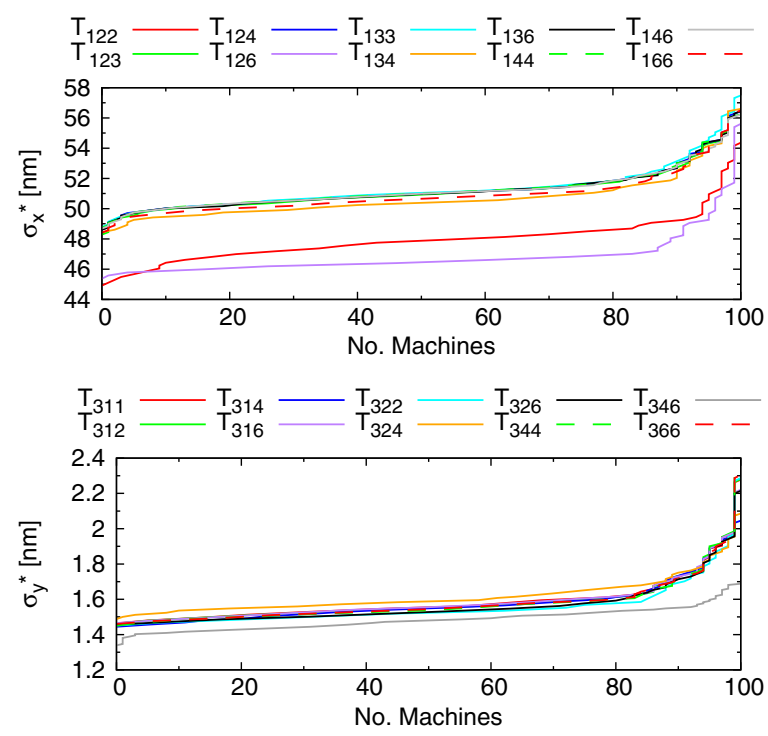

FIG. 1. Cumulative histograms over 100 IP beam distributions of $\sigma_{x}^{*}$ (top) and $\sigma_{y}^{*}$ (bottom), when the second order correlations are individually removed.

in [18], are obtained to target the mentioned linear aberrations. Additional dispersion-knobs (DFS-knobs), constructed by means of the available dipole correctors, are also obtained by decomposing the dispersion response matrix using $S V D$-analysis. Only the first four singular values are found to significantly reduce the beam size at the IP. The sextupole-based and DFS-knobs are iteratively scanned against luminosity until no further improvement in terms of luminosity is observed. At this point the IP beam distributions are analyzed. First and second order correlations are computed for each IP beam distribution. Corrected $\sigma_{x, y}^{*}$ are obtained after removing the computed correlations one at a time. It should be noted that the corrected $\sigma_{x, y}^{*}$ are obtained as the width of a Gaussian curve fitted to the corrected particle distribution, so called the core beam size, which is equal or less than the rms beam size. These analysis allows us to identify the most dominant aberrations present at the current stage of the tuning process and thus construct the required knobs to be included into the tuning procedure based on luminosity measurements. Figure 1 shows the histograms of corrected beam size when individually subtracting the second order correlations. No beam size reduction is observed when removing the linear correlations from the IP beam distributions, which means that the linear knobs are effectively working. In contrast, when removing some of the second order aberrations, a noticeable beam size reduction is clearly observed for almost all machines, as shown by the lower curves of top and bottom plots of Fig. 1. The aberrations that reduce $\sigma_{x}^{*}$ (top) and $\sigma_{y}^{*}$ (bottom) the most are: $T_{126}, T_{122}, T_{346}$ and $T_{322}$. which are defined as,

$$
T_{i, j, k}=\frac{\left\langle u_{i}, u_{j}, u_{k}\right\rangle}{\sigma_{u_{i}} \sigma_{u_{j}} \sigma_{u_{k}}}
$$
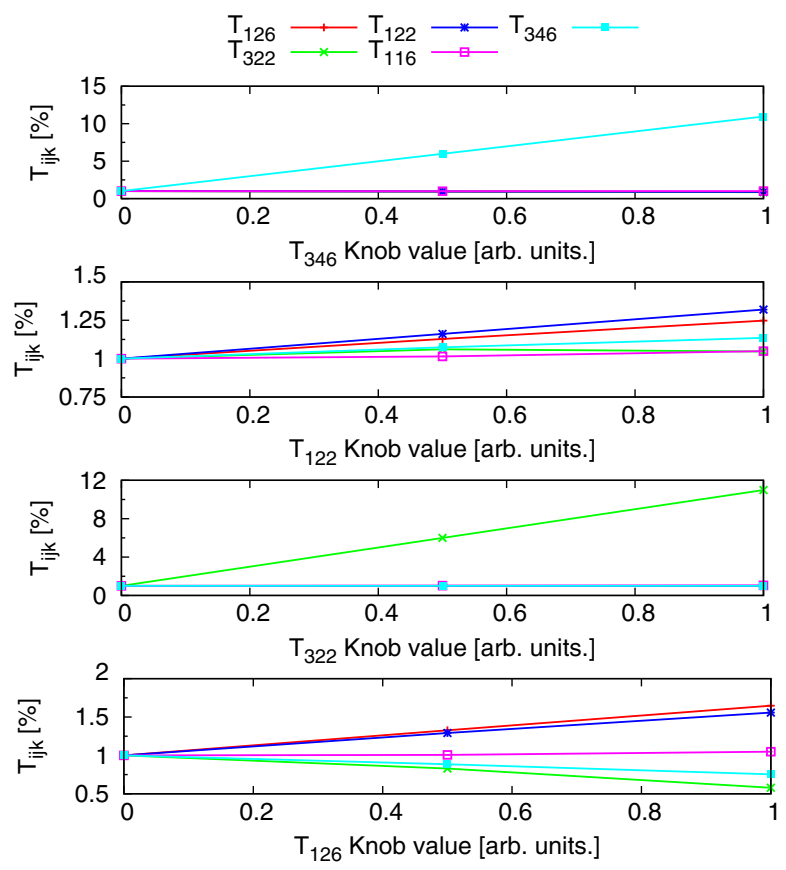

FIG. 2. Variation of $T_{126}$ (red), $T_{322}$ (green), $T_{122}$ (blue) and $T_{346}$ (cyan) correlations when scanning the $T_{126}, T_{322}, T_{122}$ and $T_{346}$ knobs from bottom to top plots.

where $u_{i, j, k}$ can be $x, x^{\prime}, y^{\prime}, y^{\prime}$ or $\frac{\Delta p}{p}$. It becomes clear that 2 ndorder knobs that target these aberrations are required in the tuning procedure to further improve the tuning performance.

Strength variations of the normal sextupole magnets present in the FFS of CLIC are used to construct tuning knobs that target the $T_{126}, T_{122}$ and $T_{346}$ aberrations. In contrast the $T_{322}-\mathrm{knob}$ is based on strength variations of skew sextupole magnets. Indeed 4 skew sextupole magnets are inserted in the $e^{-}$and $e^{+}$CLIC-FFS lattices, following the same criteria as discussed in [19]. The system response when scanning the second order knobs one-by-one is shown in Fig. 2. Knobs $T_{126}$ and $T_{122}$ are not orthogonal to the other knobs which compromises their effectiveness. Scanning the knobs one-by-one could eventually lead to no beam size reduction or luminosity gain, as one aberration gets corrected at the expenses of exciting others.

Scanning the second order knobs leads to the excitation of linear correlations that again need to be corrected by scanning the linear knobs. Therefore each set of linear and nonlinear knobs are iteratively scanned until convergence is achieved. After we analyze the IP beam distributions to figure out the effectiveness of the knobs and the remaining aberrations. One discovers that the $3 \mathrm{rd}$ order aberration $U_{3222}$, becomes relevant as the first and second order correlations are significantly minimized. To target this high-order aberration, the octupole magnet present in the vicinity of the final doublet quadrupoles, last quadrupole pair before the IP, is employed. The performance achieved by applying this tuning procedure is discussed in Sec. II C 1. 


\section{Double beam}

The tuning procedure for the double beam case is slightly modified with respect to the single beam case, to account for 2 independent systems. First, after independently applying the BBA algorithms described in Sec. II B 1, both beamlines are moved in the transverse plane, as explained in [20]. Second, the linear and nonlinear knobs obtained for the single case are scanned first on the $e^{-}$ beamline and after on the $e^{+}$one. The so-called feed-down effect from the offset sextupoles, perturb the orbit leading to a relative offset at the IP between the $e^{-}$and $e^{+}$beams, as shown in Fig. 3. The beam-beam IP feedback [21] foreseen at CLIC would correct for the observed offsets. Instead of modeling the IP feedback in our simulations, the IP $\Delta x$ and $\Delta y$-offsets are removed from the tracked distributions before evaluating the luminosity. Modeling the feedback as a dynamic system is out of the scope of this paper devoted to static imperfections.

\section{Results}

In the following, the results obtained for single and double beam studies are discussed. The results are shown in form of cumulative histograms, since our goal is set for $90 \%$ of the machines.

\section{Single beam}

In the single beam case, the luminosity is computed by assuming that $e^{-}$and $e^{+}$are identical systems, in other words, only one beam is tracked through the FFS and the obtained IP beam distribution is assumed to be exactly the same for the other beam, leading to no relative offset between the beams.

Figure 4 shows the accumulated histogram of luminosities, obtained for 100 machines, at every tuning scan. A tuning scan consists on scanning one set of knobs, either linear or nonlinear. The first curve (red) corresponds to the scan after applying $B B A$ correction algorithms. By iteratively optimizing the linear knobs, $90 \%$ of the machines

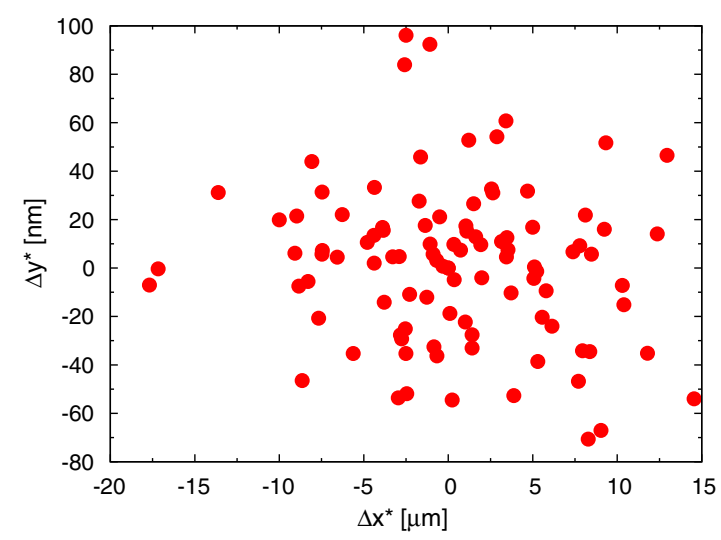

FIG. 3. Relative beam position offsets at the IP.

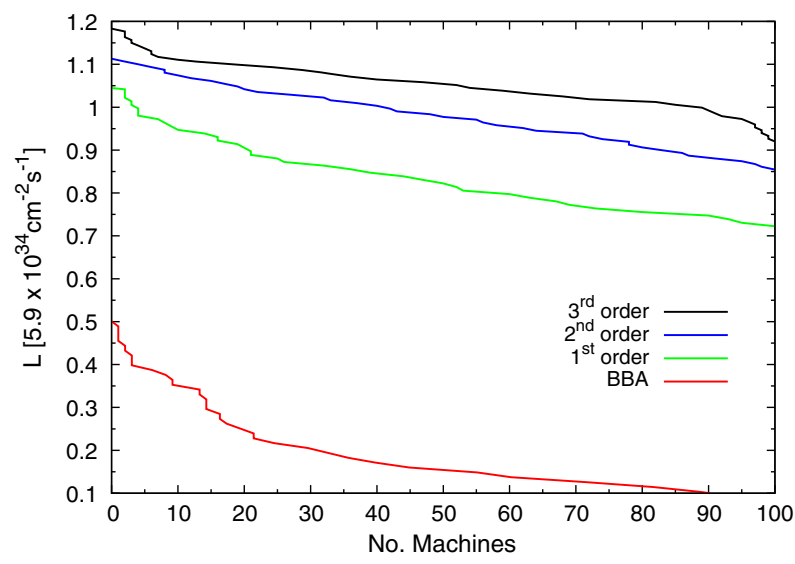

FIG. 4. Tuning results for single beam case study. Red curve shows the accumulated luminosity histogram after BBA. Green, blue, and black curves represent the maximum luminosity obtained by scanning 1st, 2nd, and 3rd order knobs, respectively.

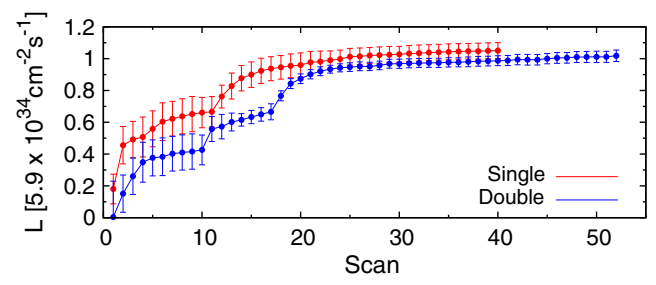

FIG. 5. Tuning convergence for single (red) and double (blue) beam studies. Each dot is the mean value of 100 machines, while the error bar is the standard deviation at each scan.

reach almost $\geq 80 \%$ of $\mathcal{L}_{0}$, as shown by the green curve. At this point no further improvement is observed by additional scans of the linear knobs. It is worth mentioning that about $20 \%$ of the machines required repeating 1 -to-1 and $B B A$ steps. For these machines the initial $B B A$ step does not bring the system to a state from which the machine fully benefits from scanning the linear knobs.

Scanning the 2nd order knobs boosts the luminosity up to $\geq 95 \%$ of $\mathcal{L}_{0}$ for $90 \%$ of the machines (blue curve). To further improve, the $U_{3222}-\mathrm{knob}$ is included in the tuning procedure, and it impact is reflected by the blue curve. By ceaselessly going over all knobs, we managed to bring $90 \%$ of the machines to a $\mathcal{L} \geq 102 \%$ of $\mathcal{L}_{0}$, as shown by the black curve. The total number of luminosity measurements is of the order of 6000. Figure 5 shows the evolution of the mean and standard deviation of the luminosity for the single beam case (red) up to 40 scans (6000 luminosity measurements). The abrupt change at scan 11 is due to the optimization of the second order knobs. Tuning convergence is much faster at the initial scans, whereas it becomes a slow process after the 20th scan due to multiple contributions of linear and nonlinear aberrations to the IP beam size. 


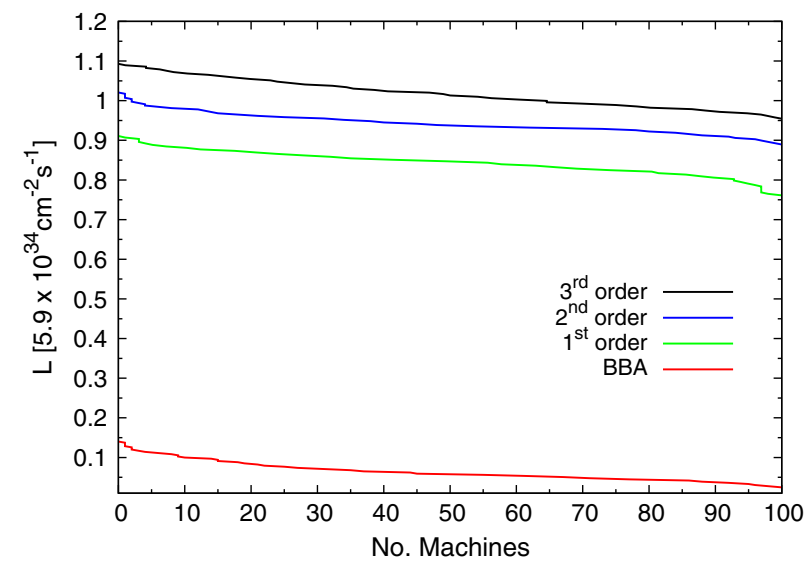

FIG. 6. Tuning results for the double beam study. Red curve shows the accumulated luminosity histogram after BBA. Green, blue and black curves represent the maximum luminosity obtained by scanning 1st, 2nd, and 3rd order knobs, respectively.

\section{Double beam}

In this case the $e^{-}$and $e^{+}$systems are treated independently. The number of luminosity measurements is going to be at least double with respect to the single beam case since the knobs cannot be simultaneously scanned for both $e^{-}$and $e^{+}$systems, otherwise one would not know which beam is shrinking in size since the luminosity is our tuning observable.

Figure 6 shows the accumulated luminosity histogram obtained for 100 machines after every scan. The 4 curves labeled on the plot correspond to the same steps as described in the single beam case. By optimizing iteratively the linear knobs, $90 \%$ of the machines reach $\geq 79 \%$ of $\mathcal{L}_{0}$. Optimization of 2nd order knobs boosts the luminosity of $90 \%$ of the machines to $\geq 92 \%$ of $\mathcal{L}_{0}$. Finally, optimization of $U_{3222}$-knob increases $\mathcal{L} \geq 97 \%$ of $\mathcal{L}_{0}$ for $90 \%$ of the machines. 52 scans are required to reach $97 \%$ of $\mathcal{L}_{0}$. The total number of luminosity measurements is of the order of 15000 . Figure 5 shows the evolution of the mean value of luminosity for the double beam case (blue) for the 52 scans. Although convergence has not been achieved, it is also noticeable that the gain provided at each scan is small, thus the tuning becomes a slow but steady process.

\section{Discussion}

As expected the realistic tuning study performs slightly worse than the simplified case in terms of luminosity and speed. The different performances in luminosity could be overpass by additional scans, since convergence has not yet been achieved in both studies, although the tuning becomes a slow and high computational consuming process at the later stage.

Regarding the tuning speed, the realistic study requires 15000 measurements to reach the same $\mathcal{L}$ performance as the single case does in only 9000 measurements, which represents a tuning speed $40 \%$ slower. One possible explanation could be the extra luminosity obtained when colliding the beam with itself.

Reference [22] shows that the luminosity scales as

$$
\mathcal{L} \approx \frac{1}{\sqrt{\sigma_{x, e^{-}}^{* 2}+\sigma_{x, e^{+}}^{* 2}} \sqrt{\sigma_{y, e^{-}}^{* 2}+\sigma_{y, e^{+}}^{* 2}}}
$$

when colliding beams of different sizes, where $\sigma_{x, y, e^{-}}^{*}$ and $\sigma_{x, y, e^{+}}^{*}$ represent the transverse electron and positron beam sizes, respectively. Assuming that $\sigma_{e^{-}}<\sigma_{e^{+}}$in both planes, relation (2) can be expressed as

$$
\mathcal{L} \approx \frac{1}{\sigma_{x, e}^{*} \sigma_{y, e}^{*} \sqrt{1+k_{x}^{2}} \sqrt{1+k_{y}^{2}}}
$$

by defining $k_{x} \equiv \frac{\sigma_{x, e^{+}}^{*}}{\sigma_{x, e^{-}}^{*}}$ and $k_{y} \equiv \frac{\sigma_{y, e^{+}}^{*}}{\sigma_{y, e^{*}}^{*}} . k_{x}$ and $k_{x}$ can only take values $\leq 1$. The equality takes place when both beams are exactly the same, reducing relation $(3)$ to $\approx \frac{1}{2 \sigma_{x, e}^{*} \sigma_{y, e}^{*}}$. In order to maximize the $\mathcal{L}$ it is desirable to keep $\tilde{x}$ as small as possible. Thus the knobs should always be applied to the largest beam size. In our study we have not taken this conclusion into account, as the knobs where scanned one at a time first on the electron beamline and later on the positron one. The beamline scan order should be considered into future simulations as it could potentially improve the tuning speed up to a factor 2 if the values are kept as small as possible throughout the tuning procedure.

Determining the largest beam size between the lepton beams could be done by using a beam size monitor of the photon beams emitted by the beamstrahlung effect, located somewhere in the machine-detector interface.

\section{CONCLUSIONS}

The CLIC-FFS tuning study has made a significant progress since the Conceptual Design Report (CDR) publication [1] in terms of performance, speed and considered imperfections. Transverse alignments and rotations imperfections of the system components, BPM reading errors and magnet strength errors have been included into simulations, bringing the study into a more realistic scenario. A better understanding of the beam aberrations, allowed us design a better tuning procedure which effectively targets the most common aberrations at the IP.

For the simplified single beam study, the number of luminosity measurements required for tuning $90 \%$ of the machines at a luminosity $\geq 102 \%$ of $\mathcal{L}_{0}$, is of the order of 6000 , which is a factor 3 times faster and a $10 \% \mathcal{L}$ increase with respect to the CDR results. 
For the realistic case where the $e^{-}$and $e^{+}$beamlines are treated independently, the obtained luminosity of $90 \%$ of the machines is equal or larger than $97 \%$ of $\mathcal{L}_{0}$ after 15000 luminosity measurements. The 5\% difference in performance with respect to the single beam case might be bridged by additional knob scans, since convergence is not yet achieved. Regarding the tuning speed, faster convergence could be achieved by scanning the knobs always on the largest particle IP beam size. Reduction of the number of $\mathcal{L}$ measurements might be achieved if photon beam sizes monitors can be integrated for both systems, in order to keep the ratio between the electron and positron beam sizes at the IP, as close as possible to 1 throughout the tuning procedure.

Looking into next studies, dynamic imperfections would be the following errors to be included into simulations. In order to minimize its impact, it would be beneficial to improve the tuning speed. Along this line, a fast luminosity measurement with a resolution of $1 \%$ or better is required, therefore identification of an effective luminosity signal would be needed. In conclusion, the study has not achieved the $110 \%$ of $\mathcal{L}_{0}$ goal for 100 different CLIC-FFS machines. At this moment best tuning results are 13\% short in luminosity and yet dynamic imperfections are not included. Given that the tuning goal was set to $110 \%$ of $\mathcal{L}_{0}$ to allow margin for dynamic imperfections. It becomes natural to extend the study by including dynamic imperfections, as ground motion, and evaluate the final performance of the CLIC-FFS.

\section{APPENDIX: LUMINOSITY RESOLUTION}

The error of the luminosity calculation depends on the number of particles employed in the calculation. In order to estimate it, 100 different bunches with different bunch populations, ranging from $10^{4}$ to $10^{5}$ particles, have been tracked through the CLIC-FFS without considering any imperfections. Results are shown in Fig. 7. In our study the number of particles used for tuning is $10^{5}$ particles for both beams, thus the error is below $1 \%$.

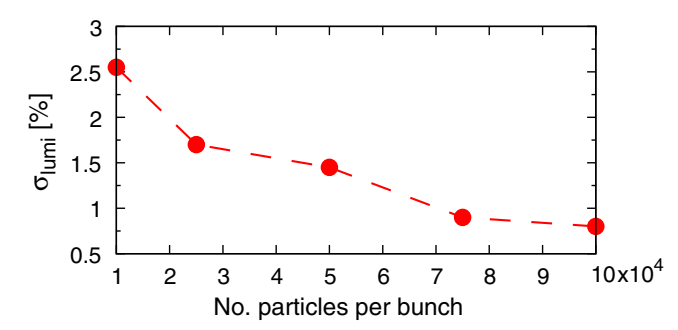

FIG. 7. Obtained luminosity error assuming an error-free CLIC-FFS lattice as a function of number of particles in the bunch. Each dot is the standard deviation of 100 luminosity calculations obtained by GUINEA-PIG after tracking 100 different bunches.
[1] P. Lebrun, L. Linssen, A. Lucaci-Timoce, D. Schulte, F. Simon, S. Stapnes, N. Toge, H. Weerts, and J. Wells, The CLIC programme: Towards a staged $e^{+} e^{-}$linear collider exploring the terascale: CLIC conceptual design report, arXiv:1209.2543.

[2] R. Tomás et al., in Proceedings of the 23rd Particle Accelerator Conference, Vancouver, Canada, 2009 (IEEE, Piscataway, NJ, 2009), p. WE6PFP024.

[3] P. Raimondi and A. Seryi, Novel final focus design for future linear colliders, Phys. Rev. Lett. 86, 3779 (2001).

[4] The ILC Collaboration, Tech. Report No. ILC-REPORT2007-001, 2007.

[5] C. Adolphsen et al., The International Linear Collider Technical Design Report-Volume 3.I: Accelerator R\&D in the Technical Design Phase, arXiv:1306.6353.

[6] G. White et al. (ATF2 Collaboration), Experimental Validation of a Novel Compact Focusing Scheme for Future Energy-Frontier Linear Lepton Colliders, Phys. Rev. Lett. 112, 034802 (2014).

[7] B. Dalena et al., Beam delivery system tuning and luminosity monitoring in the Compact Linear Collider, Phys. Rev. Accel. Beams 15, 051006 (2012).

[8] J. Snuverink, R. M. Bodenstein, and R. Tomás, Two-beam tuning in the CLIC-BDS, in Proceedings of International Particle Accelerator Conference (IPAC'16), Busan, Korea (JACoW, Geneva, 2016), p. 3508.

[9] CLICSIM Repository, https://svnweb.cern.ch/cern/wsvn/ clicsim/trunk/CLIC/Lattices/MainBeam/BDS/v_10_10_11/.

[10] D. Schulte, The tracking code PLACET, https://clicsw.web .cern.ch/clicsw/.

[11] D. Schulte, Ph.D. thesis, DESY, Hamburg, Germany, 1997.

[12] R. P. Brent, in Algorithms for Minimization without Derivatives (Prentice-Hall, Englewood Cliffs, NJ, 1973), chap. 3-4.

[13] E. Beutler and R. Mead, A Simplex method for function minimization, Computer Journal (UK) 7, 308-313 (1965).

[14] R. Assmann, P. Raimondi, G. Roy, and J. Wenninger, Emittance preservation with dispersion free steering at LEP, Phys. Rev. ST Accel. Beams 3, 121001 (2000).

[15] T. Raubenheimer, and R.D. Ruth, A dispersion free trajectory technique for linear colliders, Nucl. Instrum. Methods Phys. Res., Sect. A 302, 191 (1991).

[16] T. Raubenheimer et al., SLAC Report No. SLAC-TN-03071, 2004.

[17] A. Latina, P. Raimondi et al., in Proceedings of the 25th International Linear Accelerator Conference, LINAC-2010, Tsukuba, Japan (KEK, Tsukuba, Japan, 2010), p. 109.

[18] E. Marin Lacoma, Ph.D. thesis, CERN, 2012.

[19] T. Okugi et al., Linear and second order optics corrections for the KEK accelerator test facility final focus beam line, Phys. Rev. Accel. Beams 17, 023501 (2014).

[20] G. White, BDS BBA tuning and beam dynamics, LET Beam Dynamics Workshop at SLAC, USA (2007), http:// ilcagenda.linearcollider.org/materialDisplay.py?contribId=20 \&sessionId=11\&materialId=slides\&confId=2364.

[21] J. Pfingstner, J. Snuverink, and D. Schulte, Ground motion optimized orbit feedback design for the future linear collider, Nucl. Instrum. Methods Phys. Res., Sect. A 703, 168 (2013).

[22] W. Herr and B. Muratori, Concept of luminosity (2006), https://cds.cern.ch/record/941318.

Correction: The fifth sentence of the abstract contained an error in wording and has been corrected. 